
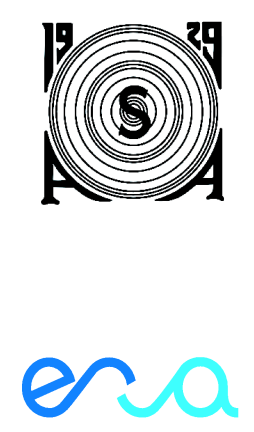

\section{Studying converted waves in shallow marine environment}

N. El Allouche ${ }^{\mathrm{a}}$, G. g Drijkoningen ${ }^{\mathrm{a}}$, W. Versteeg ${ }^{\mathrm{b}}$ and D. G Simons ${ }^{\mathrm{a}}$

${ }^{a}$ Delft University of Technology, P.O. Box Postbus 5048, 2600 GA Delft, Netherlands ${ }^{\mathrm{b}}$ Ghent University, Krijgslaan 281, S8, 9000 Ghent, Belgium

n.elallouche@tudelft.nl 
Waves converted from compressional to shear mode have been used in hydrocarbon exploration for the imaging and characterization of the deep subsurface. Since shear-waves propagate with a velocity that is dependent on the shear modulus and are thus directly related to the shear strength of the sediment, they are very useful for geotechnical purposes. In this abstract, we investigate the feasibility of using converted waves in shallow marine unconsolidated sediments to obtain S-wave information. The main focuses are on understanding the dependence of mode conversion on the seismic properties and on determining the appropriate field configuration to acquire these waves. The conversion at the water bottom is maximal at two angles where the smallest angle appears to be more favorable in the environment of interest. However, the amplitude of this conversion is much less than that at an interface between two sediment layers. Numerical experiments show that placing the receivers at the water bottom (OBC-configuration) or in a borehole is required to observe converted waves. Another main result is that the waves converted only once at a reflector and recorded at a post-critical angle have the best chance to be observed on a seismogram.

\section{Introduction}

For several decades, waves converted from compressional to shear mode have been successfully used in hydrocarbon exploration to image and characterize the subsurface. These converted waves have proven to be useful in cases where conventional $\mathrm{P}$-waves provide poor results due to the low acoustic impedance contrast or the presence of shallow gas in the pores. The usefulness of this method arises from the fact that $\mathrm{S}$-waves, in contrast to $\mathrm{P}$-waves, propagate with a velocity that is dependent on the shear modulus and are thus directly related to the shear-strength properties of the sediment. In the last three decades, various authors, have shown that the S-wave velocity is much more sensitive to changes in lithology and mechanical properties than the Pwave velocity. At the shallow marine scale, the determination of these properties is important for geotechnical purposes.

In general, P-wave reflection amplitudes as a function of offset, called AVO in geophysical exploration are inverted to retrieve S-wave information. However, Riedel et al. [1] showed that this approach does not always give satisfactorily results for a shallow marine environment. The large uncertainty in the $\mathrm{S}$-wave velocity estimation is ascribed to the insensitivity of the P-wave amplitude to this seismic parameter. This insensitivity is caused by the relatively low seismic velocity contrasts for this type of environment.

An alternative approach is to obtain S-wave information from converted waves. However, it is not clear whether these waves can be used for geotechnical aims. In this modelling study, we investigate the feasibility of acquiring converted waves in shallow marine unconsolidated sediments. The main objectives are to understand the dependence of mode conversion on the seismic properties and to determine the appropriate field setting to record this type of waves.

In this paper, we study the conversion coefficients at the water bottom and at an interface between two sediment layers to assess their dependence on the angle of incidence and the seismic parameters of the medium. Furthermore, we compute the seismic response expected from a horizontally layered medium in different field configurations and we conclude by comparing the effective amplitude of the converted waves to the pure P-waves.

\section{Conversion coefficients}

In the marine case, an incident $\mathrm{P}$-wave can be converted to an $\mathrm{S}$-wave at the water bottom or at a sediment/sediment interface. Conversion at both types of interfaces is studied to assess which mode may be important for detection of shear-wave information in a shallow marine setting.

\subsection{Conversion at water bottom}

At a plane water bottom, an incident P-wave is reflected, transmitted or converted to S-wave (Figure 1a). As is well known, the conversion coefficients at a fluid-solid interface are obtained from requiring the continuity of normal components of displacement and traction and the vanishing of shear component of traction. Then, the conversion transmission coefficient $T_{P S}$, defined as the displacement amplitude ratio of the transmitted S- and incident P-wave, is adapted from [1]

$$
T_{p s}=\frac{2 \cos \theta_{1}}{\left(\cos \theta_{1}\left(A_{1}-A_{2} \frac{\rho_{2} \alpha_{2}}{\rho_{1} \alpha_{1}} \cos \left(2 \varphi_{2}\right)\right)-A_{2} \cos \left(2 \theta_{2}\right)+\sin \left(\varphi_{2}\right)\right)},
$$

with

$A_{1}=\frac{\rho_{2} \alpha_{2} /\left(\rho_{1} \alpha_{1}\right)}{\alpha_{2} / \beta_{2}} \sin \left(2 \varphi_{2}\right)$ and $A_{2}=\frac{\alpha_{2}}{\beta_{2}} \frac{\cos \left(2 \varphi_{2}\right)}{\sin \left(2 \theta_{2}\right)}$,

where $\alpha, \beta$ and $\rho$ denote respectively the P-wave velocity, the $\mathrm{S}$-wave velocity and the density of the medium. The corresponding angles are plotted in figure $1 \mathrm{a}$.

Figure $1 \mathrm{~b}$ shows the variation of the P-SV converted transmission coefficient $T_{P S}$ with the angle of incidence. Seismic parameters typical to shallow marine environment are considered. The reflected $R_{P P}$ and transmitted $T_{P P}$, both normalized by the incident $\mathrm{P}$-wave, are included in Figure $1 \mathrm{~b}$ for comparison.

In this specific example, the conversion from $\mathrm{P}$ - to S-mode is maximal at an angle between 40 and 50 degrees, zero at the critical angle and reaches a maximum value again at an angle larger than 80 degrees. The effects of increasing the $\mathrm{P}$-wave velocity of the sediment, from 1550 to $1750 \mathrm{~m} / \mathrm{s}$, are shown in Figure 2a. It results in a decrease of the critical angle. The maximum conversion at the post-critical angle increases at the expense of the conversion at the smaller angle. Furthermore, it can be observed in Figures $2 b$ and $2 c$ that changes in S-wave velocity and density affect the amplitude of the conversion coefficients but not so much the location of the maximum. 


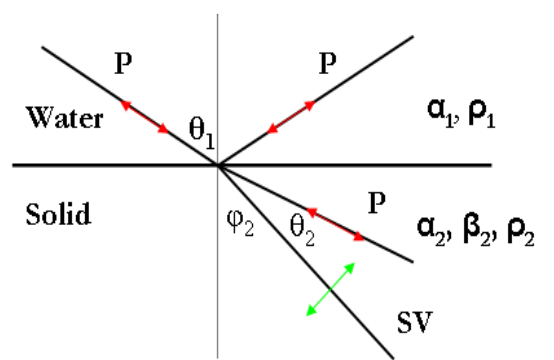

(a)

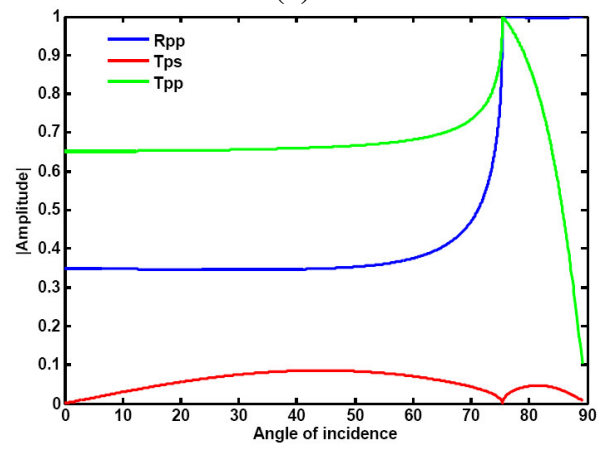

(b)

Figure 1: a) Sketch of the energy partitioning at a waterbottom interface $b$ ) reflection, transmission and conversion coefficients calculated for the model parameters: $\alpha_{1}=1500$ $\mathrm{m} / \mathrm{s}, \rho_{1}=1000 \mathrm{~kg} / \mathrm{m}^{3}, \alpha_{2}=1550 \mathrm{~m} / \mathrm{s}, \beta_{2}=200 \mathrm{~m} / \mathrm{s}$ and $\rho_{2}=2000 \mathrm{~kg} / \mathrm{m}^{3}$.

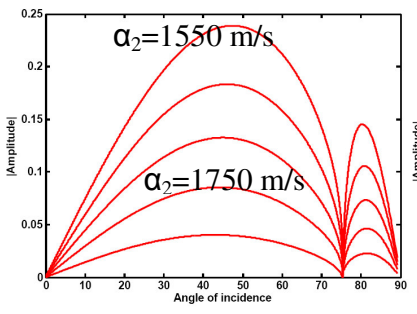

(a)

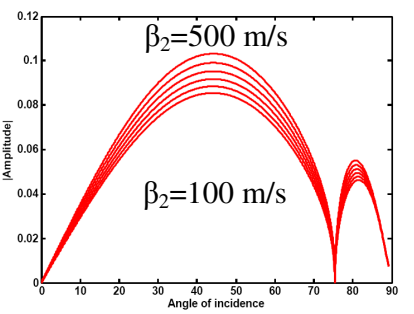

(b)

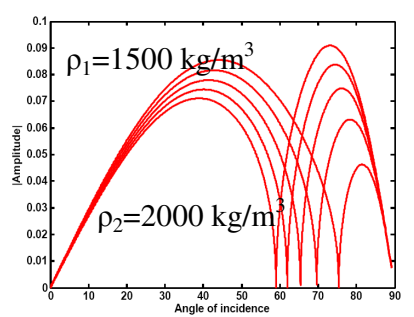

(c)

Figure 2: Normalized amplitude of $T_{P S}$ as a function of incident angle for varying: a) $P$-wave velocity $b$ ) $S$-wave velocity and c) density.

\subsection{Conversion at a sediment/sediment interface}

The energy partitioning at a welded contact between two sediment layers is depicted in Figure 3a. From the Zoeppritz equations [2] the following expression for the reflection conversion coefficient $R_{P S}$ is obtained from Aki and Richards [3]
$R_{p s}=\frac{-2 \frac{\cos \theta_{1}}{\alpha_{1}}\left(a b+c d\left(\cos \theta_{1} / \alpha_{1}\right)\left(\cos \varphi_{2} / \beta_{2}\right)\right) \sin \theta_{1}}{\beta_{1}(E+F)}$

with

$$
\begin{aligned}
& E=\left(b \frac{\cos \theta_{1}}{\alpha_{1}}+c \frac{\cos \theta_{2}}{\alpha_{2}}\right)\left(b \frac{\cos \varphi_{1}}{\beta_{1}}+c \frac{\cos \varphi_{2}}{\beta_{2}}\right) \\
& F=\frac{\sin ^{2} \theta_{1}}{\alpha_{1}^{2}}\left(a-d \frac{\cos \theta_{1}}{\alpha_{1}} \frac{\cos \varphi_{2}}{\beta_{2}}\right)\left(a-d \frac{\cos \theta_{2}}{\alpha_{2}} \frac{\cos \varphi_{1}}{\beta_{1}}\right)
\end{aligned}
$$

in which

$$
\begin{gathered}
a=\rho_{2}\left(1-2 \beta_{2}^{2} \sin ^{2} \theta_{1} / \alpha_{1}^{2}\right)-\rho_{1}\left(1-2 \beta_{1}^{2} \sin ^{2} \theta_{1} / \alpha_{1}^{2}\right) \\
b=\rho_{2}\left(1-2 \beta_{2}^{2} \sin ^{2} \theta_{1} / \alpha_{1}^{2}\right)-2 \rho_{1} \beta_{1}^{2} \sin ^{2} \theta_{1} / \alpha_{1}^{2} \\
c=\rho_{1}\left(1-2 \beta_{1}^{2} \sin ^{2} \theta_{1} / \alpha_{1}^{2}\right)-2 \rho_{2} \beta_{2}^{2} \sin ^{2} \theta_{1} / \alpha_{1}^{2} \\
d=2\left(\rho_{2} \beta_{2}^{2}-\rho_{1} \beta_{1}^{2}\right)
\end{gathered}
$$

The dependence of the transmission and reflection coefficients on the angle of incidence is given in Figure $3 \mathrm{~b}$. The P-SV converted reflection coefficient $R_{P S}$ shows a trend similar to $T_{P S}$. At moderate angles, remarkably, more energy is reflected as an $\mathrm{S}$-wave than as a $\mathrm{P}$-wave. The effect of varying the seismic parameters of the lower medium on $R_{P S}$ is shown in Figure 4 . It can be noticed that a small contrast in $\mathrm{S}$-wave velocity causes the convergence of the two maxima to a single peak at the critical angle.

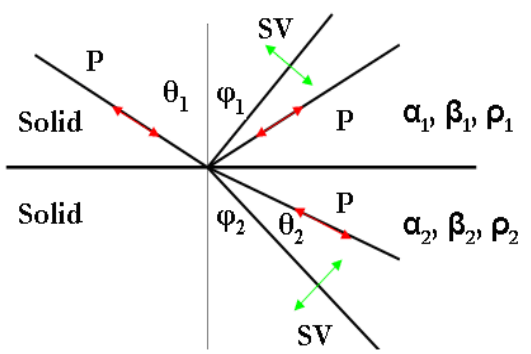

(a)

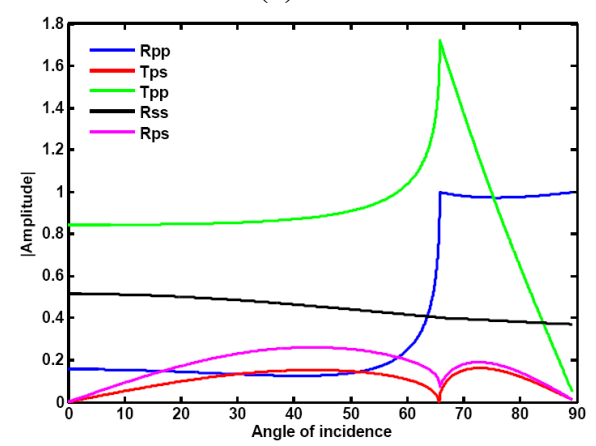

(b)

Figure 3: a) Sketch of the energy partitioning at a waterbottom interface $b$ ) reflection, transmission and conversion coefficients for model parameters: $\alpha_{1}=1550 \mathrm{~m} / \mathrm{s}$, $\rho_{1}=1000 \mathrm{~kg} / \mathrm{m}^{3}, \beta_{1}=200 \mathrm{~m} / \mathrm{s}, \alpha_{2}=1700 \mathrm{~m} / \mathrm{s}, \beta_{2}=500 \mathrm{~m} / \mathrm{s}$ and $\rho_{2}=2500 \mathrm{~kg} / \mathrm{m}^{3}$.

From the conversion coefficients, we expect P-waves transmitted at the water-bottom and then converted to $\mathrm{S}$ waves at a reflector (PPS) to have the highest amplitude. 
Down-going compressional waves converted at the water bottom and reflected at an interface (PSS) are likely to be observed on a seismogram due to the relatively large shear wave reflection coefficient $R_{S S}$ at a wide range of angles (Figure 3b).

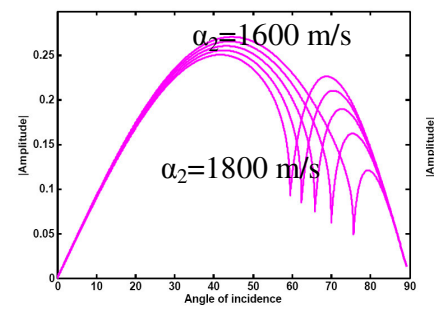

(a)

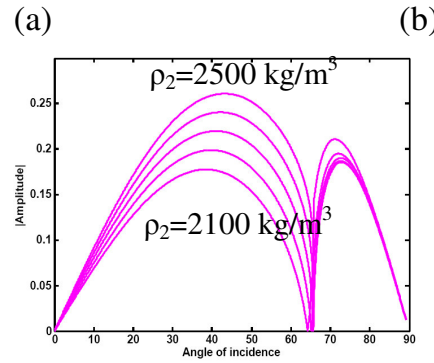

(c)

Figure 4: Normalized amplitude of $R_{P S}$ as a function of incident angle for varying: a) $P$-wave velocity $b$ ) $S$-wave velocity and c) density.

\section{Converted waves in different configurations}

To further investigate where and how converted waves can be recognized in field data, we compute the seismic response for a simple model representative of the studied environment. The model consists of a $20 \mathrm{~m}$ water layer and a $20 \mathrm{~m}$ unconsolidated sediment layer overlying a bedrock.

The conversion-coefficient results from the previous section emphasize the necessity of multi-channel recording. Therefore we compute the response for three field configurations commonly used in multi-channel seismic surveying for hydrocarbons. The modelling is performed using an elastic time-domain finite-difference algorithm which includes all propagation modes but excludes anelasticity. The source wavelet selected is a Gaussian with a peak frequency of $300 \mathrm{~Hz}$.

\subsection{Hydrophone-streamer configuration}

A hydrophone streamer consists of a string of sensors which records the pressure below the water surface. The field configuration and the primary conversion modes expected are depicted in figure $5 \mathrm{a}$. The distance between the hydrophones is $2 \mathrm{~m}$ and the source is located $7.5 \mathrm{~m}$ below the surface.

To be able to identify the various seismic arrivals we also compute the corresponding travel time using simple raytrace modelling as shown in Figure 5b. Roughly speaking, the reflections follow hyperbolic shapes known as the normal move-out. This shape is dependent on the rootmean-square propagation velocity of the event.
The seismogram as shown in Figure $5 \mathrm{c}$ is dominated by multiples. As a consequence, converted waves are difficult to observe in this configuration. After applying a windowed energy normalization known as automatic gain control (AGC), the PS-SP conversion mode becomes weakly visible at far offset. This is due to its low root-mean-square velocity and as a consequence divergent hyperbolic shape. The stronger arrival at $0.25 \mathrm{~s}$ is an interbed multiple.

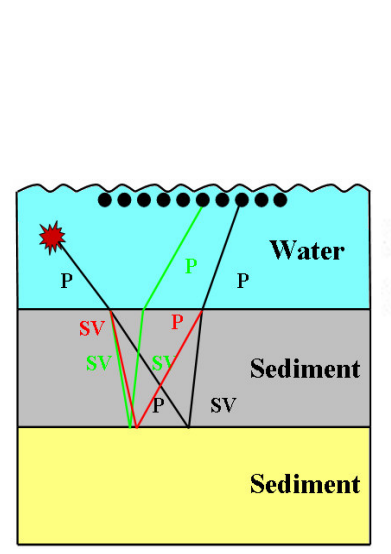

(a)

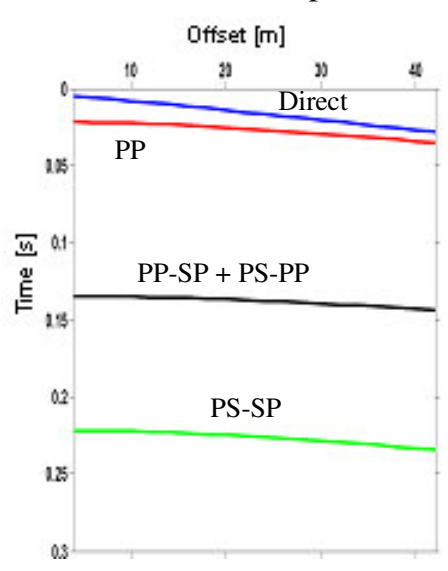

(b)

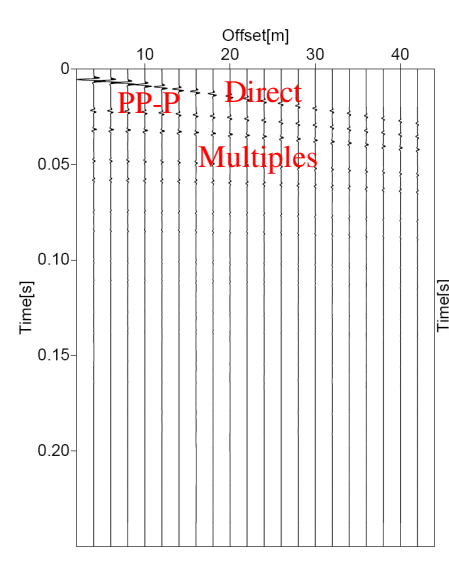

(c)

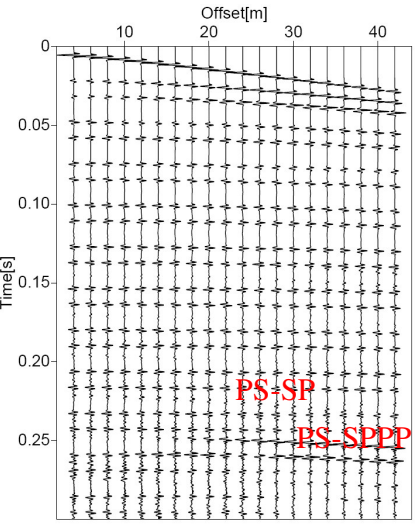

(d)
Figure 5: a) Sketch of the three layered geological model. Upper sediment layer: $\alpha_{1}=1550 \mathrm{~m} / \mathrm{s}, \rho_{1}=2000 \mathrm{~kg} / \mathrm{m}^{3}$,

$\beta_{1}=200 \mathrm{~m} / \mathrm{s} ;$ lower sediment layer: $\alpha_{2}=1700 \mathrm{~m} / \mathrm{s}$, $\beta_{2}=500 \mathrm{~m} / \mathrm{s}$ and $\rho_{2}=2500 \mathrm{~kg} / \mathrm{m}^{3}$. b) Travel time of main arrivals. c) Pressure recorded near the surface. d) Pressure after applying $A G C$.

\subsection{Water-bottom configuration}

In hydrocarbon exploration, ocean-bottom cables (OBC) are widely used to acquire converted waves at the water bottom. Such a cable contains three-component sensors measuring the particle velocity at the water-bottom and hydrophones measuring the pressure at the same level. The converted waves are expected to have the highest amplitude in the horizontal component since shear waves have a predominantly horizontal motion. Another advantage of measuring in the horizontal direction is that multiples are not so strongly present. The seismogram in Figure 6 shows clearly the presence of a superposition of two converted modes namely the PP-S and the PS-P. As expected from the conversion coefficients, the PS-S converted wave can also be identified. 


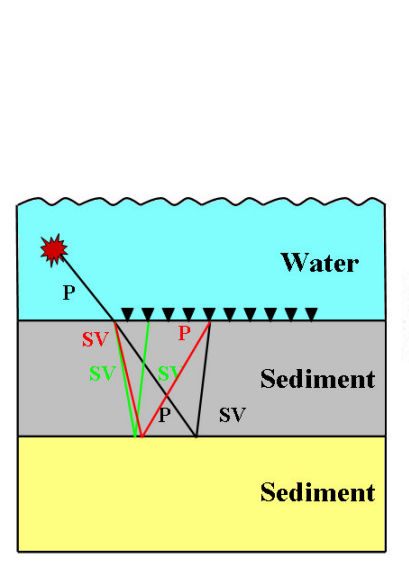

(a)

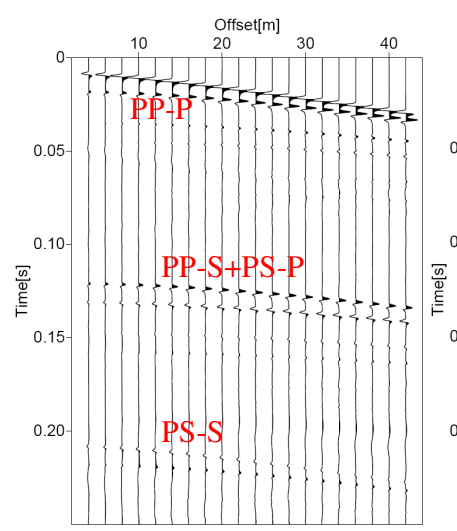

(c)

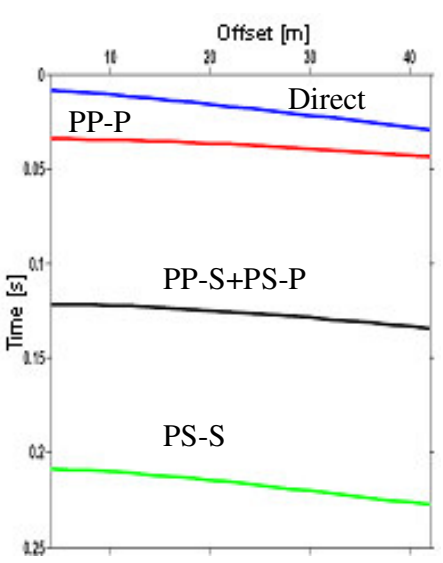

(b)

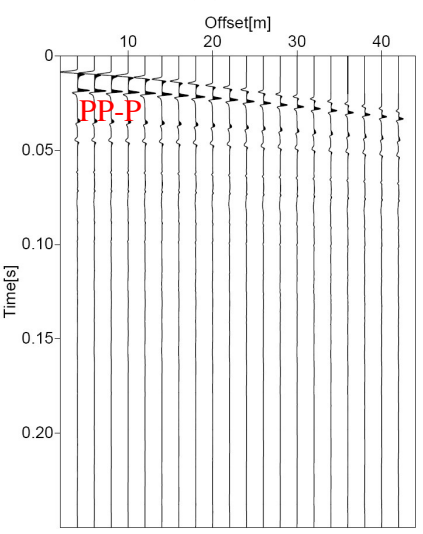

(d)
Figure 6: a) Sketch of the three layered geological model. Upper sediment layer: $\alpha_{1}=1550 \mathrm{~m} / \mathrm{s}, \rho_{1}=1000 \mathrm{~kg} / \mathrm{m}^{3}, \beta_{1}$ $=200 \mathrm{~m} / \mathrm{s} ;$ lower sediment layer: $\alpha_{2}=1700 \mathrm{~m} / \mathrm{s}, \beta_{2}=500$ $\mathrm{m} / \mathrm{s}$ and $\rho_{2}=2500 \mathrm{~kg} / \mathrm{m}^{3}$. b) Travel time of main arrivals. c) Particle velocity recorded in the horizontal direction.

d) Particle velocity recorded in the vertical direction.

\subsection{Borehole configuration}

In this configuration (Figure 7), three-component geophones are placed vertically in a well. From figures $8 \mathrm{a}$ and $8 \mathrm{~b}$, it can be noticed that different converted modes are well observed in the horizontal component and absent in the vertical which makes this setting very appropriate in distinguishing between the purely compressional waves and the converted ones.

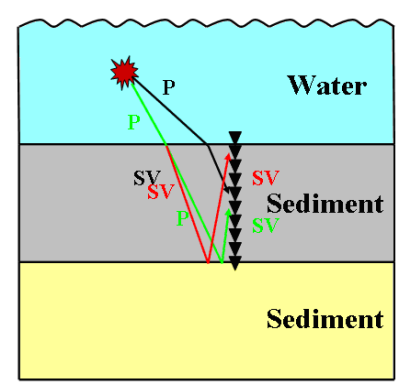

Figure 7: Sketch of the borehole configuration. The distance between the receivers is $2 \mathrm{~m}$. The source is at a depth of $7.5 \mathrm{~m}$.

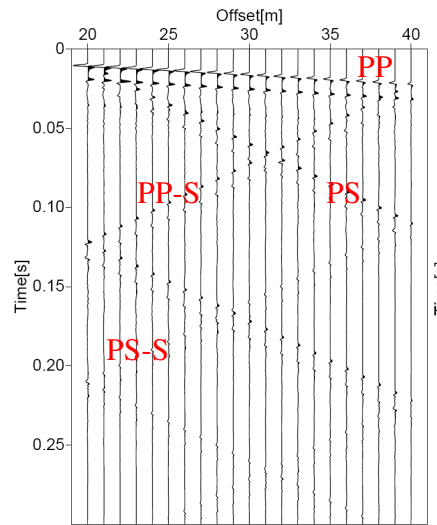

(b)

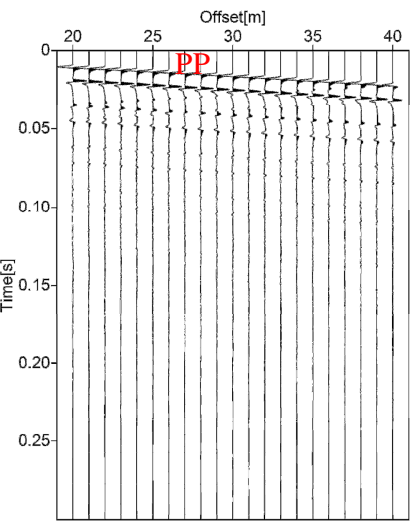

(c)
Figure 8: a) Sketch of the three layered geological model. Upper sediment layer: $\alpha_{1}=1550 \mathrm{~m} / \mathrm{s}, \rho_{1}=2000 \mathrm{~kg} / \mathrm{m}^{3}, \beta_{1}$ $=200 \mathrm{~m} / \mathrm{s} ;$ lower sediment layer: $\alpha_{2}=1700 \mathrm{~m} / \mathrm{s}, \beta_{2}=500$ $\mathrm{m} / \mathrm{s}$ and $\rho_{2}=2500 \mathrm{~kg} / \mathrm{m}^{3}$. b) Particle velocity recorded in the horizontal direction. c) Particle velocity recorded in the vertical direction.

\section{$4 \quad$ Multi-layered model}

In practice, many types of converted modes can be generated in the subsurface but not all of them carry enough energy to be visible on a seismic record. In this section we attempt to assess which modes of conversion have the potential to be observed by computing their effective amplitude and comparing it to that of the pure P-waves.

We distinguish two categories of mode conversion namely the reflected-converted and the transmitted-converted waves. In order to include more types of converted waves than the ones discussed above we complicate our model by adding another $20 \mathrm{~m}$ thick sediment layer in between with a $\mathrm{P}$-wave velocity of $1650 \mathrm{~m} / \mathrm{s}$, S-wave velocity of $300 \mathrm{~m} / \mathrm{s}$ and a density of $2200 \mathrm{~kg} / \mathrm{m}^{3}$. Again we calculate the fullwave response for an OBC-configuration.

As shown in Figure 9, many events are visible in the computed seismograms. These are identified using a raytracing program. Note that symmetrical modes have the same travel time. This is only the case in a horizontally layered medium.

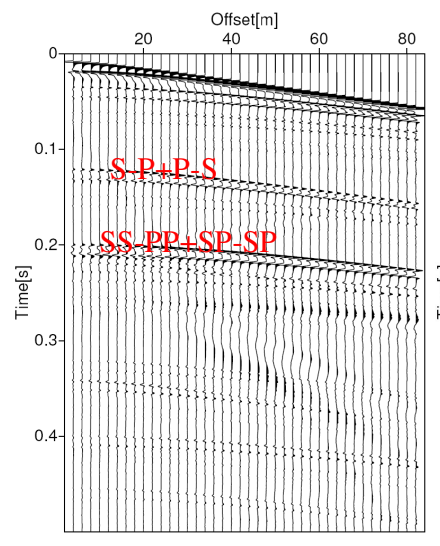

(a)

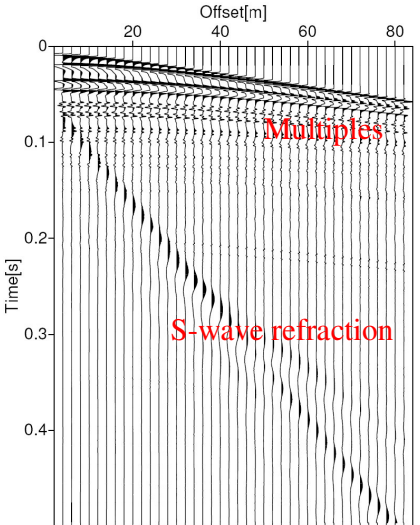

(b)
Figure 9: Synthetic seismogram for multi-layered sediment model. a) Particle displacement in horizontal direction. b) Particle displacement in vertical direction. 
The obtained seismogram can numerically be decomposed by taking the divergence and the rotation of the computed wavefield. The results given in Figure 10 show a decrease of the P-wave energy in the horizontal component, such that more converted events become observable. These weaker events are transmitted-converted waves which travelled most of their path as an S-wave.

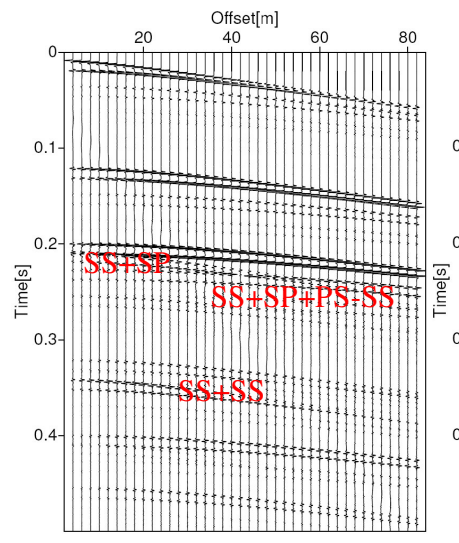

(a)

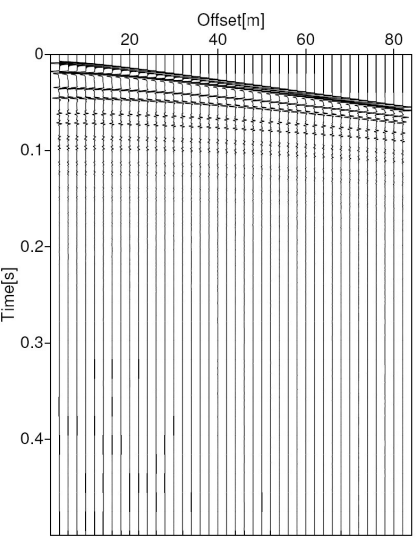

(b)
Figure 10: Synthetic seismogram for multi-layered sediment model. Particle displacement after decomposition in horizontal direction (a) and vertical direction (b).

The amplitude is affected by geometrical spreading and the energy partitioning at an interface. To examine only the effect of the latter, we calculate the effective reflection coefficients of the observed arrivals, consisting of the product of all transmission and reflection coefficients encountered by the waves in their travel path. The amplitude of the effective reflection coefficients are computed for the events observed on the seismograms. Note that the amplitude of the converted waves is much weaker than that of the P-reflections.

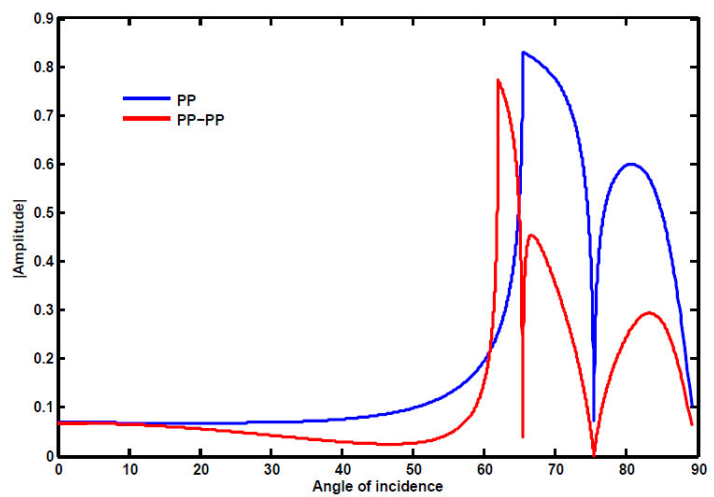

Figure 11: Effective reflection coefficients for the PP, PP$P P$.

The effective reflections of the strongest transmittedconverted and reflected-converted events are displayed in Figure 12 and 13 respectively. Clearly, waves converted only once at a reflector provide the best chance to be observed at post-critical angle.

\section{Conclusions}

We have shown via a modelling study how we could obtain shear-wave information in a shallow marine environment. At the water-bottom most energy is expected to be transmitted as P-waves. However, at the sediment/sediment interface and for the considered model parameters, more energy seems to be reflected as an S-wave than as a $\mathrm{P}$ wave. The conversion is maximal at two angles where the smallest angle seems to be more favourable in the case of unconsolidated sediment with relatively low $\mathrm{P}$-wave velocity. Acquiring converted waves requires the use of multi-channel recording therefore three configurations considered common in seismic surveying were modelled. The water-bottom and the borehole configurations are appropriate for obtaining shear-wave information via converted-waves. In the ocean-bottom configuration, waves converted only once at a sediment reflector and recorded at post-critical angle are expected to be the strongest modes. For future work, the considered model can be extended to a more realistic representation of the shallow marine environment including anelastic effects and the results will be validated with field experiments.

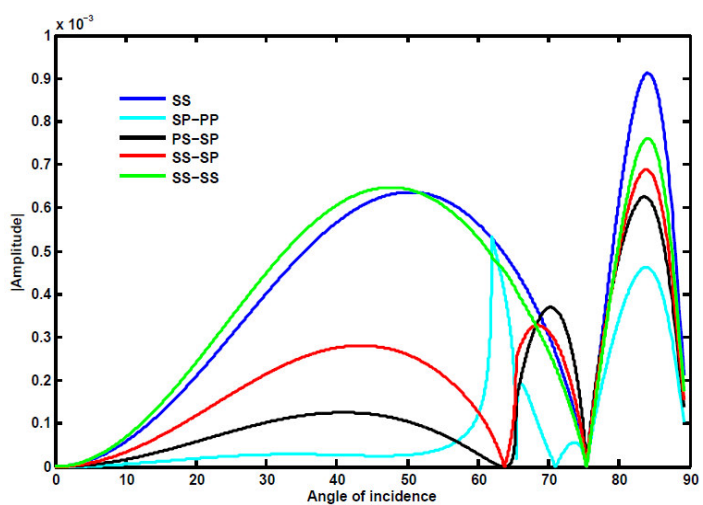

Figure 12: Effective reflection coefficients for the strongest transmitted-converted waves.

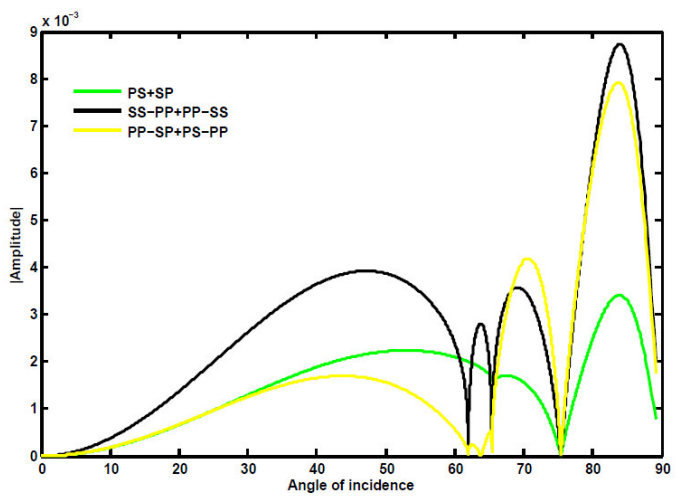

Figure 13: Effective reflection coefficients for the strongest reflected-converted waves.

\section{References}

[1] K. Aki and P. G. Richards, "Quantitative Seismology Theory and Methods", W. H. Freeman, Vol.1. (1980)

[2] M. Riedel and F. Theilen, "AVO investigations of shallow marine sediments ", Geophysical Prospecting 49, 198-212 (2001)

[3] K. Zoeppritz, "Erdbebenwellen VIII B On the reflection and penetration of seismic waves through unstable layers", Goettinger Nachr., 66-84. 